\title{
Coronagraphic imaging of three weak-line T Tauri stars: evidence of planetary formation around PDS 70^
}

\author{
P. Riaud ${ }^{1}$, D. Mawet ${ }^{1}$, O. Absil ${ }^{1}$, A. Boccaletti ${ }^{2}$, P. Baudoz ${ }^{2}$, E. Herwats ${ }^{1,3}$, and J. Surdej ${ }^{1}$ \\ 1 IAGL, Université de Liège, 17 Allée du 6 Août, 4000 Sart Tilman, Belgium \\ e-mail: riaud@astro.ulg.ac.be \\ 2 LESIA, Observatoire de Paris-Meudon, 5 pl. Jules Janssen, 92195 Meudon, France \\ ${ }^{3}$ Laboratoire d'Astrophysique de l'Observatoire de Grenoble, BP 53, 38041 Grenoble Cedex 9, France
}

Received 20 March 2006 / Accepted 20 June 2006

\section{ABSTRACT}

\begin{abstract}
Context. High angular resolution imaging of nearby pre-main sequence stars with ages between 1 and 30 Myr can give valuable information on planet formation mechanisms. This range of ages is thought to correspond to the dissipation of the optically thick dust disks surrounding young stars and to the end of the planet formation.

Aims. This paper presents new observations of three weak-line T Tauri Stars (WTTS) of intermediate ages ranging from 7 to $16 \mathrm{Myr}$. It aims at increasing the knowledge and sample of circumstellar disks around "old" WTTS.

Methods. We observed three stars with the VLT's NAOS-CONICA adaptive optics system in coronagraphic mode. The four-quadrant phase mask coronagraph was used to improve the dynamic range (by a factor of $\sim 100$ ) while preserving the high angular resolution (inner working angle of $0 . ' 15$ ).

Results. One object of our sample (PDS 70), a K5 star, exhibits a brown dwarf companion and a disk in scattered light with a surface brightness power law of $r^{-2.8}$, extending from a distance of 14 to $140 \mathrm{AU}$ (assuming a stellar distance of $140 \mathrm{pc}$ ) and an integrated luminosity of $16.7 \mathrm{mJy}$ in the $K_{\mathrm{s}}$-band. The mass of the companion can be estimated to be within a range between 27 and 50 Jupiter masses with an effective temperature of $2750 \pm 100 \mathrm{~K}$. This object also shows a resolved outflow stretching up to $250 \mathrm{AU}$.

Conclusions. This newly detected circumstellar disk shows strong similarities with the disk around TW Hya, and adds to the observed population of "old" TTS surrounded by circumstellar material. Moreover, three clues of planetary formation are brought to light by this study.
\end{abstract}

Key words. stars: planetary systems: protoplanetary disks - stars: circumstellar matter - instrumentation: adaptive optics methods: observational

\section{Introduction}

Three different periods of star formation are generally distinguished. Young stars like those in Taurus and Chamaeleon (1-3 Myr) are embedded in their cocoon emitting only in the far-infrared and millimeter wavelengths. Older stars like Vega (350 Myr) or Fomalhaut (200 Myr) show large dissipated debris disks residing between 50 and $150 \mathrm{AU}$. In between, we find objects of intermediate age like $\beta$ Pic or Au Mic (20 Myr), which are surrounded by disks of gas and dust still in the process of forming planets.

The study of objects between 1 and $30 \mathrm{Myr}$ is therefore of special importance to constrain the planet formation scenario. Moreover, previous works indicating that the 1 to $10 \mathrm{Myr}$ period is likely to be the timescale for disk dissipation (e.g., Haisch et al. 2001; Mosqueira \& Estrada 2006) have to be confirmed by further observations in this age range. When the gas reservoir is dissipated and the accretion stops, class III stars are left with planetesimals, potentially continuing planet formation in a more tenuous disk. The most natural explanation for the presence of planets is that the growth from micron-sized dust to planetesimals is extremely efficient. Once planetesimals grow beyond the $\mathrm{km}$-size, runaway accretion is thought to drive those that are far

* Based on observations obtained with NACO/FQPM at the Paranal Observatory, Chile, in ESO program 075.C-0730(A). enough from the central star to planetary size. The alternative scenario of gravitational instability, proposed by Boss (2002), could also be at the origin of planet formation around low and intermediate mass stars.

The main observable feature at these early formation stages is the general morphology of the disk (brightness profile, asymmetries, etc.) from which timescales for disk accretion, dissipation, and planet building can be inferred, as we will discuss. To better understand these phenomena, one needs to increase the number of observations of young and intermediate objects. A classical reservoir for young stars is the TW Hydra association, which contains various PMS stars. Only three optically thick disks have been detected so far in young associations: firstly around TW Hya (Weinberger et al. 2002; Krist et al. 2000), GM Aurigae (Schneider et al. 2003), and recently around PDS 144 (Perrin et al. 2006). Other young stellar associations exist in our neighbourhood $(<150 \mathrm{pc})$, such as Centaurus, Lupus, or Ophiuchus, and their observation is actually far from being completed.

In this paper, we present the results of coronagraphic observations of three PMS stars (T Tauri) with ages comprised between 7 and 16 Myr (in Scorpius, Corona Australis, and Centaurus). These observations were performed in the nearinfrared $K_{\mathrm{s}}$-band with the VLT's NACO adaptive optics system during 3 nights from the 22th to the 24th of June 2005. One star 
Table 1. VLT/NACO observing log of 7 stars in the $K_{\mathrm{s}}$-band with the FQPM coronagraph.

\begin{tabular}{|c|c|c|c|c|c|c|c|c|c|c|}
\hline Target & $\alpha(\mathrm{J} 2000)$ & $\bar{\delta} \delta(\mathrm{J} 2000)$ & UT date & Exposure time & $\overline{\text { DIT }}$ & Seeing & $\overline{\tau_{0}^{*}(m s)}$ & Airmass & Strehl & Astigmatism \\
\hline PDS 70 & $14: 08: 10$ & $-41: 23: 53$ & $22 / 07 / 2005$ & $1500 \mathrm{~s}$ & $5 \mathrm{~s}$ & 1."4 & 1.1 & 1.1 & $20 \% \pm 3 \%$ & $134^{\circ} \pm 2^{\circ}$ \\
\hline HBC609 $9^{\text {ref }}$ & 15:59:16 & $-41: 57: 10$ & $22 / 07 / 2005$ & $900 \mathrm{~s}$ & $5 \mathrm{~s}$ & $1^{\prime \prime} 7-2$."7 & $1-0.8$ & 1.05 & $15 \% \pm 6 \%$ & $131^{\circ} \pm 2^{\circ}$ \\
\hline PDS 70 & 14:08:10 & $-41: 23: 53$ & $23 / 07 / 2005$ & $1140 \mathrm{~s}$ & $5 \mathrm{~s}$ & 0.9 & 1.5 & 1.1 & $32 \% \pm 3 \%$ & $130^{\circ} \pm 2^{\circ}$ \\
\hline TTS $18^{\text {ref }}$ & $15: 14: 47$ & $-42: 20: 14$ & $23 / 07 / 2005$ & $1080 \mathrm{~s}$ & $5 \mathrm{~s}$ & 0.9 & 1.6 & 1.1 & $35 \% \pm 2 \%$ & $128^{\circ} \pm 2^{\circ}$ \\
\hline PDS 81 & 16:14:08 & $-19: 38: 28$ & $24 / 07 / 2005$ & $2200 \mathrm{~s}$ & $5 \mathrm{~s}$ & $1^{\prime \prime}$ & 1.8 & 1.05 & $30 \% \pm 9 \%$ & $130^{\circ} \pm 2^{\circ}$ \\
\hline HIC $89529^{\text {ref }}$ & 18:16:07 & $-18: 37: 03$ & $24 / 07 / 2005$ & $2200 \mathrm{~s}$ & $3 \mathrm{~s}$ & $1^{\prime \prime}$ & 1.8 & 1.1 & $35 \% \pm 8 \%$ & $131^{\circ} \pm 2^{\circ}$ \\
\hline PDS 99 & 19:09:46 & $-37: 04: 26$ & $24 / 07 / 2005$ & $960 \mathrm{~s}$ & $4 \mathrm{~s}$ & $1^{\prime \prime}$ & 1.8 & 1.3 & $28 \% \pm 10 \%$ & $122^{\circ} \pm 2^{\circ}$ \\
\hline $\mathrm{SS} 300^{\mathrm{ref}}$ & 17:18:08 & $-38: 08: 27$ & $24 / 07 / 2005$ & $960 \mathrm{~s}$ & $4 \mathrm{~s}$ & 1."1 & 1.4 & 1.4 & $30 \% \pm 7 \%$ & $128^{\circ} \pm 2^{\circ}$ \\
\hline
\end{tabular}

${ }^{*} \tau_{0}$ corresponds to the atmospheric correlation time at $0.5 \mu \mathrm{m}$ recorded during the observation.

ref Reference stars.

of our sample (PDS 70, a K5 star) presents a large disk and a jet-like structure. The detection of the disk in scattered light was possible thanks to the four-quadrant phase-mask (FQPM) coronagraph (Rouan et al. 2000; Riaud et al. 2001). This new generation coronagraphic device allows both good stellar extinction and high angular resolution imaging.

In Sect. 2, we describe the targets, the data analysis procedure, and the associated observational artefacts, taking the effect of the FQPM coronagraph into account. In Sect. 3, we present the $K_{\mathrm{s}}$-band observation results. Section 4 is then dedicated to the discussion of the general properties of the PDS 70 disk. Two complementary numerical models are introduced for that purpose. They show that the presence of a young cold dust disk under dissipation reproduces the observed disk characteristics and sheds new light on former thermal infrared data.

\section{Observations and data analysis}

\subsection{Observations}

Observations were performed with the VLT's NAOS-CONICA adaptive optics system (NACO) in the coronagraphic mode, using the FQPM coronagraph. This phase mask coronagraph uses a four quadrant $\pi$ phase-shift distribution in the focal plane to provide an efficient destructive interference of the on-axis star. The FQPM coronagraph has been validated on a test bench in monochromatic light (Riaud et al. 2003) showing peak attenuation of $\sim 10^{5}$, and recently, in polychromatic light between 500 to $900 \mathrm{~nm}$ (Mawet et al. 2006) with a peak attenuation of $\sim 750$. A monochromatic device manufactured on an infrasil substrate has been installed and commissionned on the NACO instrument (Boccaletti et al. 2004). Under good seeing conditions $\left(<0\right.$ '. $\left.^{\prime}\right)$, a peak attenuation of about 10-30 is routinely obtained, for an inner working angle of $0 . ' 15$.

A sample of young stellar objects were imaged during three nights (June 22 to 24, 2005), using the visible wavefront sensor of NACO (Rouan et al. 2000). All FQPM coronagraphic images were taken with the $K_{\mathrm{S}}$ filter and the $\mathrm{S} 13$ camera (13.27 mas/pixel). This relatively high image sampling (4 pixels per $\lambda / d$ ) allows a precise centering on the phase mask coronagraph, and therefore a good reference subtraction for the data analysis. The observing conditions are reported in Table 1. Two objects, PDS 70 and its associated reference star HBC 609, were observed under poor seeing conditions during the first night. For this reason, PDS 70 was re-observed during the second night, but with another reference star (TTS18). The two other WTTS were also observed with their proper reference star: PDS 81 with HIC 89529 and PDS 99 with SS300. It is to be noted that, to close the loop, AO wavefront sensing is directly performed in the visible on the target under acquisition, be it
Table 2. Spectral and photometric characteristics of the stars.

\begin{tabular}{cccccccc}
\hline \hline Target & Sp & $m_{V}$ & $m_{K}$ & $F_{12 \mu \mathrm{m}}^{*}$ & $3 \sigma^{\dagger}$ & $F_{25 \mu \mathrm{m}}^{*}$ & $3 \sigma^{\dagger}$ \\
\hline PDS 70 & K5 & 12.0 & 8.5 & 270 & 24 & 430 & 51.6 \\
HBC609 & K8 & 12.0 & 8.6 & $n d$ & - & $n d$ & - \\
TTS18 & K1 & 11.3 & 9.0 & $n d$ & - & $n d$ & - \\
PDS 81 & M0 & 11.8 & 7.7 & 610 & 48.8 & 1320 & 158.4 \\
HIC89529 & M1? & 11.3 & 7.5 & $n d$ & - & $n d$ & - \\
PDS 99 & M2 & 13.1 & 8.3 & 580 & 40.6 & 1430 & 85.8 \\
SS300 & M2? & 12.1 & 7.4 & $n d$ & - & $n d$ & - \\
\hline
\end{tabular}

${ }^{*}$ The flux in $12 \mu \mathrm{m}$ and $25 \mu \mathrm{m}$ in mJy is provided by the IRAS catalog (Neugebauer et al. 1988).

Photometric error in mJy. $n d$ Not detected with IRAS.

a reference star or the scientific object. It is necessary to have the same atmospheric turbulence corrections between the target and the reference star. For that, all reference stars were chosen for their similar magnitudes in the $V$ band (for similar AO correction) and $K_{\mathrm{s}}$-band (for similar a signal-to-noise ratio in the CONICA camera). Due to the need for similar colours, almost all of the observed objects are young stars (T Tauri). This could lead to some issues in circumstellar material detections, as the references are also likely to possess a disk. This eventuality has been carefully checked by looking at their thermal infrared excesses in the IRAS catalog (see Table 2).

The total integration time ranged from 900 to $2200 \mathrm{~s}$ depending on the target. To calibrate time-dependent PSF variations, we acquired reference coronagraphic images ninety minutes before or after the scientific exposures at almost the same parallactic angle. To reduce drift and pupil rotation, the target star centering was checked and corrected every $60 \mathrm{~s}$.

\subsection{Data analysis}

The sum of individual short coronagraphic images is processed in the following way. A normalized "super-flat" is created by taking the median of five lamp-flats with appropriate dark frame subtractions. The NACO coronagraphic mode requires the calibration of each target star with a corresponding median sky exposure, allowing the subtraction of the background and dark contributions. The subsequent normalization of each subtracted image with the "super-flat" provides a first stage of data processing. However, as we will see, this simple treatment will not completely prevent the presence of an electronic noise due to the readout process, as well as two electronic ghosts of the star, appearing on both sides of the detector center.

Next, all images are co-added with a sub-pixel centering process. Indeed, because of the camera sampling of 


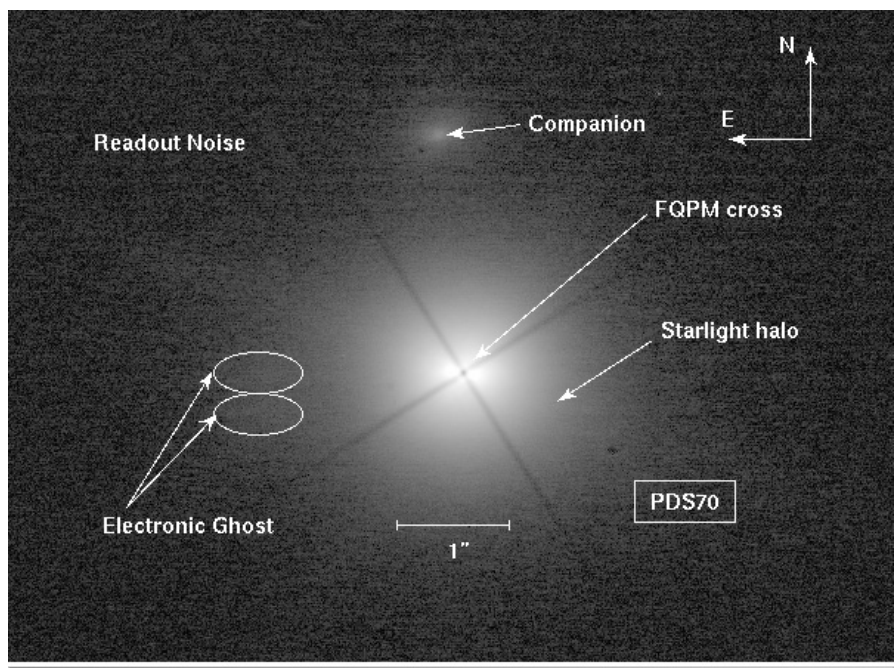

Fig. 1. Preprocessed coronagraphic image of PDS 70 obtained by the sum of 19 images (19 min of total exposure time). The FQPM cross and the dark hole in the center of the image are clearly visible. Four bright peaks are surrounded by a residual starlight halo (3"' 3 in diameter). We also show two electronic ghosts and the noise due to the readout. The logarithmic brightness scale ranges between 0 and 2000 ADU.

13.27 mas per pixel, it has been empirically demonstrated that a sub-pixel precision of 2 mas $\mathrm{rms}$ is necessary to achieve an efficient coronagraphic image addition. For this operation, a two-dimensional Gaussian fit of the dark center of the FQPM ( $F W H M$ of 60 mas) coronagraph provides the needed sub-pixel precision for the centering in the Fourier domain. Doing this, we obtained about $\sigma=0.05$ pixel or 0.7 mas rms of centering error, which is 3 times better than the specification mentioned here above. In the reduced and co-added images, the diffracted starlight appears in fact much brighter than the flux of a putative disk. Figure 1 shows the preprocessed coronagraphic image of the PDS 70 source. One can notice the classical structure of FQPM coronagraphic images: four peaks at the center surrounded by a large smooth starlight halo.

A reference star is then subtracted to increase the contrast of the coronagraphic image by minimizing the effect of the diffracted starlight smoothed halo in a field of view (FOV) of 3.'5 in diameter centered on the FQPM mask. Let $I_{\mathrm{obj}}, I_{\text {ref }}$ be the flux for the target and the reference star, respectively, in the coronagraphic image. For a proper subtraction, a classical least square procedure is applied. The corresponding figure-of-merit function $\epsilon$ corresponds to the residue of the subtraction between the target and the reference:

$\epsilon=\sum_{i, j} \frac{\left|I_{\mathrm{obj}}(i, j)-\alpha \cdot I_{\mathrm{ref}}(i, j)-\beta\right|^{2} \times S(i, j)}{\sqrt{I_{\mathrm{obj}}(i, j)}}$.

The minimization parameter $\alpha$ is the scale intensity factor for the reference image. $\beta$ corresponds to the background offset between the reference and target images. The latter is determined by taking the median value of the image parts where no significant signal is detected. The $S$ function is a pixel-varing function used to mitigate the image over-subtraction issue by increasing the weight of pixels with large deviation relative to the shot noise. For example, this parameter is set to 1 if the subtraction in the pixel $(i, j)$ is lower than three times the shot noise $\sqrt{I}_{\mathrm{obj}}$, otherwise it is set to 10 :

$S=\left\{\begin{array}{lll}10 & \text { if } & \left|\left(I_{\mathrm{obj}}-\alpha \cdot I_{\mathrm{ref}}-\beta\right)\right|>3 \sqrt{I}_{\mathrm{obj}} \\ 1 & \text { if } & \left|\left(I_{\mathrm{obj}}-\alpha \cdot I_{\mathrm{ref}}-\beta\right)\right|<3 \sqrt{I}_{\mathrm{obj}}\end{array}\right.$

In fact, tests have been carried out with the parameter $S$ ranging from 1 to 100 , and 10 is the retained trade-off value. It is to be noted that if the reference star possesses circumstellar features, it would create a false over-subtraction issue. However, we have noted that the calculus of the optimal $\alpha$ that minimizes $\epsilon$ is a robust procedure little sensitive to the exact value of $S$ and to the presence of circumstellar features around the reference.

Finally, it can be convenient to know the flux level in the pixel $(i, j)$ relative to the azimuthally median value in a crown at the distance $r=\sqrt{\left(i^{2}+j^{2}\right)}$. For that, an azimuthally averaged profile is removed from the reduced data to further enhance the contrast of imperfectly circular circumstellar features. Before this operation, it is necessary to check the image to detect the presence of perfectly circular circumstellar features. However, it is to be noted that this operation can give a negative value for data below the median profile.

\subsection{Residual noise}

The dominant source of residuals after subtraction is the speckle noise due to the variation of the turbulence conditions between the science object and the reference star. The main effect is that the resulting Strehl ratio after adaptive optics correction is different for both stars. Moreover, due to the relatively faint $V$ magnitude of our targets, the performance of the adaptive optics system is limited by the shot noise and therefore provides incomplete correction of low-order aberrations like astigmatism $\left(Z_{5}, Z_{6}\right)$, coma $\left(Z_{7}, Z_{8}\right)$, or trifoil $\left(Z_{9}, Z_{10}\right)$. Indeed, all coronagraphic images present a strong residual astigmatism that is oriented in the East direction at about $130^{\circ}$ (see Table 1), leading, after subtraction, to an important speckle pattern oriented according to the astigmatism mismatch between the target and the reference stars. Therefore, a calibration of the astigmatism for all stars has been performed on the image taken without the coronagraph. For that, we fit a 2-D elliptical Gaussian profile on the PSF image using the IDL task GAUSS2DFIT. For PDS 70, the presence of a companion allows us to calibrate this astigmatism directly in the coronagraphic image using the same procedure.

In addition to the speckle noise, all subtracted images also exhibit various residual features:

- two electronic ghosts, which can only be subtracted at the level of the shot noise $(3 \sigma)$;

- some residuals due to the spider diffraction pattern;

- a negligible dither (electronic) noise due to the read-out noise of the InSb Aladdin 3 camera of CONICA.

\section{Observations in the $K_{s}$-band}

This section is devoted to the presentation of the observation results for our three targets. First of all, PDS 81 and PDS 99 present some bright residual speckles near the center $(r<0$ '.5). These residuals are in general oriented as the residual astigmatism. This speckle structure in the final image partly prevents the detection of faint disk-like structures with angular separations smaller than $0 .{ }^{\prime} 5-1^{\prime \prime}$. 

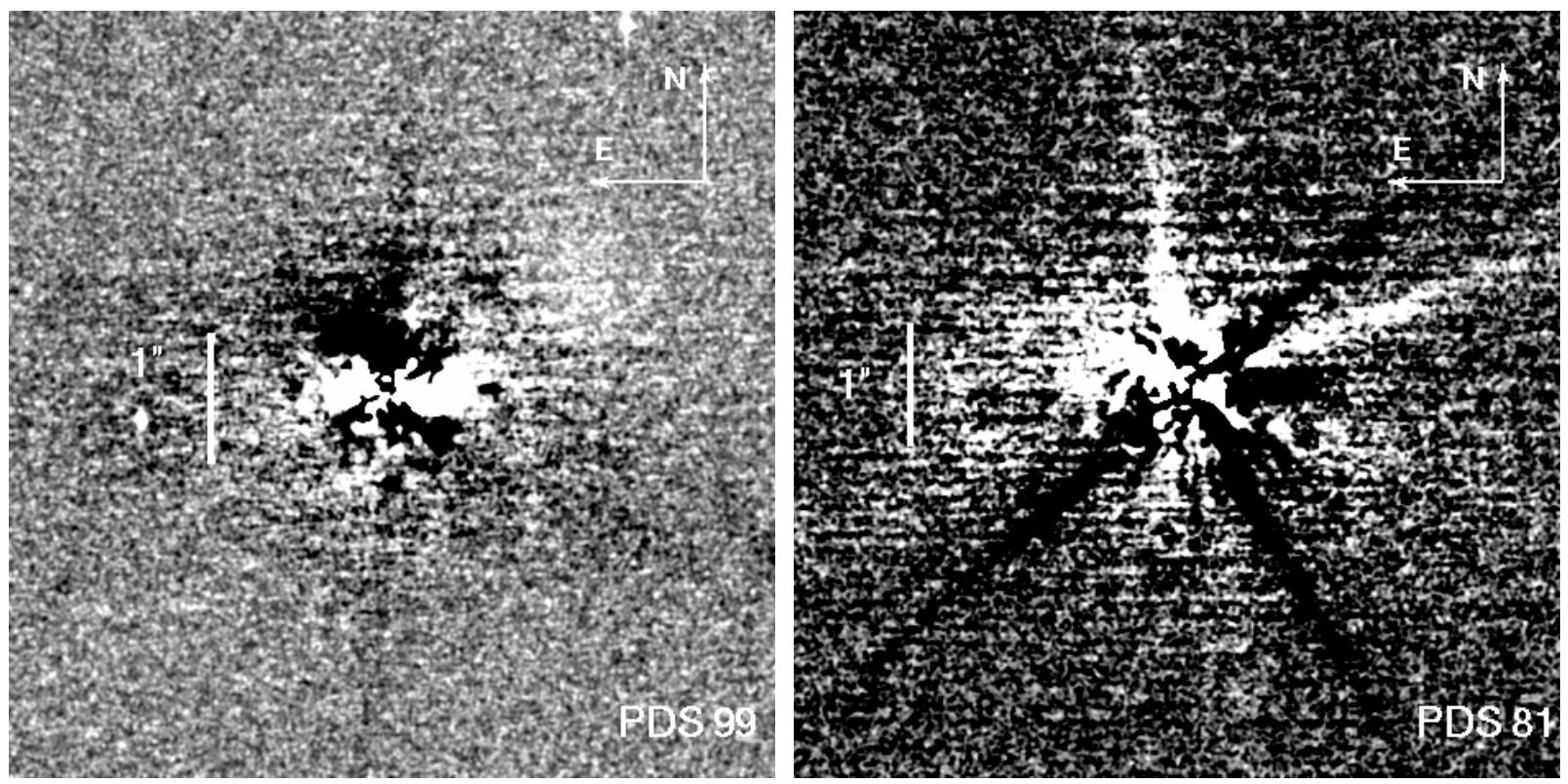

Fig. 2. Coronagraphic images: left, PDS 99 (SS300 reference star) and right, PDS 81 (HIC89529 reference star). The reference subtraction reveals bright residual speckles near the center but no large extension. For PDS 81, we can see residuals due to the differential spider rotation with respect to the reference star. The brightness scale is logarithmic.

\subsection{PDS 81 (IRAS 16112-1930) in Scorpius}

For PDS 81, the coronagraphic image after all data analysis presents residual circular features near the center (see Fig. 2). Their orientations strongly depend on the reference star used (astigmatism mismatch). No significant extended structure is detected.

\subsection{PDS 99 (IRAS 19063-3709) in Corona Australis}

For PDS 99, images were obtained with a worse seeing (1."1) than PDS 70. The final coronagraphic image presents a residual speckle pattern within $0{ }^{\prime} 5$ and oriented according to reference star astigmatism (see Fig. 2 where the reference is SS300). Again, no large-scale circumstellar feature is detected.

\subsection{PDS 70 (IRAS 14050-4109) in Centaurus}

Owing to the better adaptive optics corrections for the PDS 70 source (see Table 1), the final image presents a smaller speckle noise than for the previous targets. Table 3 summarizes the main characteristics of the star in the near- and mid-infrared. The presence of an optically thick circumstellar disk has already been suggested by the detection of a mid-infrared excess and of a strong emission in the millimeter regime (Metchev et al. 2004). In our $K_{\mathrm{s}}$-band coronagraphic data, a large disk feature in scattered light is detected as well as a companion. The disk orientation is $155^{\circ} \pm 2.5^{\circ}$ (i.e., not related to the residual astigmatism, oriented at $\left.130^{\circ} \pm 2^{\circ}\right)$.

\subsubsection{Circumstellar feature analysis}

The residual speckles from the coronagraphic image of the star are particularly sensitive to instrumental drifts. To increase the robustness of the disk detection, all reference stars (HBC 609, TTS 18, HIC 89529, SS300) were subtracted from the PDS 70 image following the data analysis procedure presented here above. Then, a median image between the four subtracted
Table 3. Main characteristics of the PDS 70 star.

\begin{tabular}{cc}
\hline \hline Age (Myr) & $<10^{\dagger}$ \\
Temperature (K) & $4406^{\dagger}$ \\
Stellar mass (solar mass) & 0.82 (K5 estimation) \\
Stellar radius (solar radius) & $1.39^{\ddagger}$ \\
Stellar luminosity (solar unit) & $0.64^{\ddagger}$ \\
Stellar luminosity (bolometric in solar unit) & $0.78^{\ddagger}$ \\
Distance (pc) & 140 \\
Temperature of the dust & $270^{\ddagger} / 45^{\ddagger}$ \\
Dust luminosity fraction $f_{\mathrm{d}}=L_{\mathrm{IR}} / L_{*}$ & $0.29^{\dagger} / 0.24^{\ddagger}$ \\
$A_{\mathrm{v}}$ & $0.74^{\dagger} / 0.81^{\ddagger}$ \\
$U / B / V$ (mag) & $14.32 / 13.15 / 12.15^{\dagger}$ \\
$R / I(\mathrm{mag})$ & $11.35 / 10.58^{\dagger}$ \\
$J / H / K(\mathrm{mag})$ & $9.55 / 8.82 / 8.54^{* *}$ \\
$N(\mathrm{mag})$ & $5.49^{*}$ \\
$60 / 100 \mu \mathrm{m}$ & $0.915 / 2.11 \mathrm{Jy}{ }^{+}$ \\
\hline
\end{tabular}

(Gregorio-Hetem \& Hetem 2002). $\$$ (Metchev et al. 2004). * (Kessler-Silacci et al. 2005). ${ }^{* *}$ (Skrutskie et al. 2006). ${ }^{+}$(Neugebauer et al. 1988).

images was derived. It must be noted that the speckle level of this final image can be determined by an analysis of the six cross subtractions between the four references. The result of this procedure is that the speckle noise can be estimated and compared with the median image of PDS 70. The last data reduction step, as mentioned in Sect. 2.2, consists of subtracting an azimuthally averaged profile. In the case of PDS 70, this leaves an important over-subtraction residual in the direction perpendicular to the disk. Figure 3 shows the median frame obtained.

The knowledge of the spider diffracted light in the final image is mandatory to calibrate the subsequent contamination. For that, we simulated adaptive optics snapshot coronagraphic images under a low Strehl ratio $(\approx 30 \%)$, taking into account pupil rotation during the exposure time. Between the first and the last exposure on PDS 70, the spider rotates by $22^{\circ}$. The corrected atmospheric turbulence was simulated with 750 phase screens (corresponding to a $1 \mathrm{~s}$ exposure time). All amplitude images 

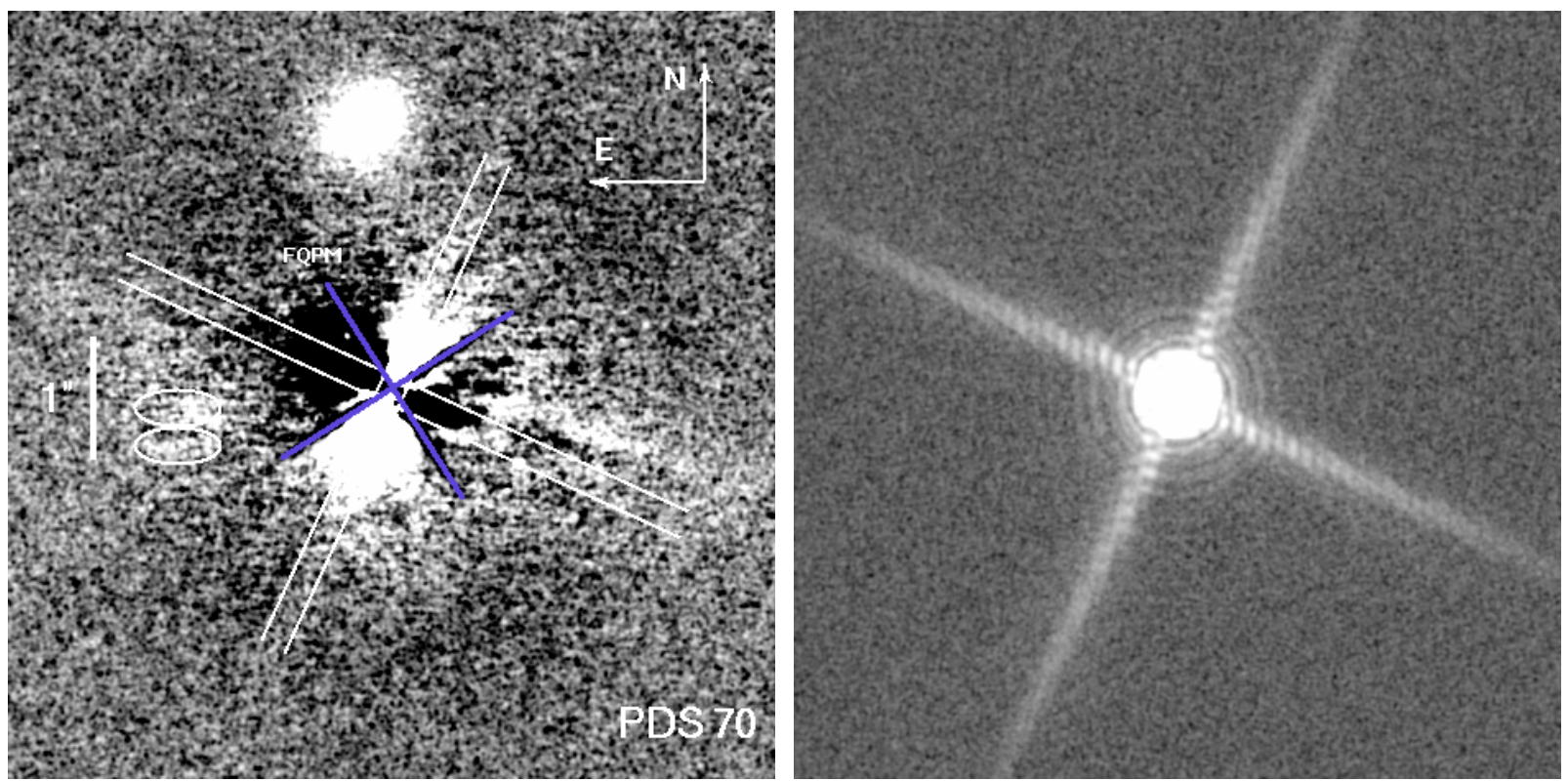

Fig. 3. Left: final image of PDS 70 star after the data analysis procedure explained in Sect. 2. The two white ellipses show the read-out ghost residuals, the white cross shows the location of the spider diffraction pattern, and the FQPM orientation is displayed in blue. Right: numerical coronagraphic model of the optical train response to calibrate the contamination due to the spider rotation (both images have the same orientation and pixel scale).

were then passed through by a monochromatic FQPM coronagraph with a working wavelength of $2.15 \mu \mathrm{m}$.

The frames produced with the 750 phase screens were coadded, rescaled (bilinear approximation), shifted, and rotated to match image size, position, and inclination. Thanks to this simulation, the contribution of the spider to the diffracted light was calibrated. This contribution comprises two main features:

- the large scale classical four arm spider diffraction pattern (Fig. 3, right);

- and an inner halo $\left(r<00^{\prime} 6\right)$ slightly brighter, but still 20 to 50 times fainter than the residual flux detected around PDS 70 (Fig. 3).

The final result (Fig. 4) shows only a weak contamination due to the spider contribution (as compared with Fig. 3, where it is not removed). For example, some insignificant artifacts at angular separations greater than $1^{\prime \prime}$ were removed.

\subsubsection{Companion analysis}

The coronagraphic image of PDS 70 shows the presence of a possible companion (PDS 70B) to the North. We do not have astrometric data to confirm its bounded character. Its $K$ magnitude is $13.29 \pm 0.02$. The $K_{\mathrm{s}}$-band photometry has been performed with the DAOPHOT package included in the IDL astrolib library. We checked the photometry accuracy with various aperture radii ranging from 10 to 80 pixels. Best results were obtained with a radius of 30 pixels for the companion and 70 pixels for the PDS 70 star. If this previously unknown companion was gravitationally linked with PDS $70(140 \mathrm{pc})$, it would correspond to a M8 stellar type located at $301.75 \pm 0.06$ AU from PDS 70A. The companion is very similar to 2M1207 A (Chabrier et al. 2000) as far as luminosity and age (also 5 to $10 \mathrm{Myr}$ ) are concerned. We have thus used the dusty models of Chabrier et al. (2000) and Baraffe et al. $(2002,2003)$ to model PDS 70B. The mass of the companion can then be estimated to be in a range between 27 and 50 Jupiter masses with an effective temperature of $2750 \pm 100 \mathrm{~K}$, both values in favor of a brown dwarf type. A confirmation that the companion is bounded would be interesting in that it would increase the population of the "brown dwarf desert" (see Matzner \& Levin 2005).

\section{Discussion}

In the following, we discuss the properties of the PDS 70 disk by interpreting our near-infrared observations (Sect. 4.1). Thermal infrared photometry data and the geometrical shape knowledge acquired provide a robust Spectral Energy Distribution (SED) fitting for this object (Sect. 4.2). Finally, the disk modeling will allow us to constrain the disk mass and extension (Sect. 4.3).

\subsection{Near infrared observations}

Disk fitting. The high dynamic range provided by the FQPM on the NACO instrument allowed the detection of the faint disk of PDS 70. The classical method for measuring the ellipticity of circumstellar disks is to calculate the radii of isophotal contours as a function of the azimuthal angle. Unfortunately, the presence of the FQPM cross imposes that we only fit isophots in the nonattenuated zones of the disk coronagraphic image (see Fig. 4). The resulting best fit for four isophots gave $e=0.466 \pm 0.025$ where $e$ is the disk ellipticity. This fit takes the previously determined disk orientation of $155^{\circ} \pm 2.5^{\circ}$ into account. If this ellipticity is interpreted as the result of the inclination of a circular disk $(\cos i)$, the measured inclination is $i=62.2^{\circ} \pm 1.6^{\circ}$. The total disk flux, measured in an annulus between 0 . $^{\prime} 05$ and $1^{\prime \prime}$ (7.4 to $140 \mathrm{AU}$ assuming $140 \mathrm{pc}$ for the distance of PDS 70) is $16.7 \pm 0.8 \mathrm{mJy}(11.49 \pm 0.05 \mathrm{mag})$ in the $K_{\mathrm{s}}$-band. The error bar is related to the photometric error, but ground-based observations under medium seeing conditions always lead to systematic uncertainties, making this value a lower bound. Indeed, the various sources of residuals add to the measured flux.

The averaged disk surface brightness in the $K_{\mathrm{s}}$-band is presented in Fig. 5. The profile is calculated between 30 and $70 \mathrm{AU}$ 


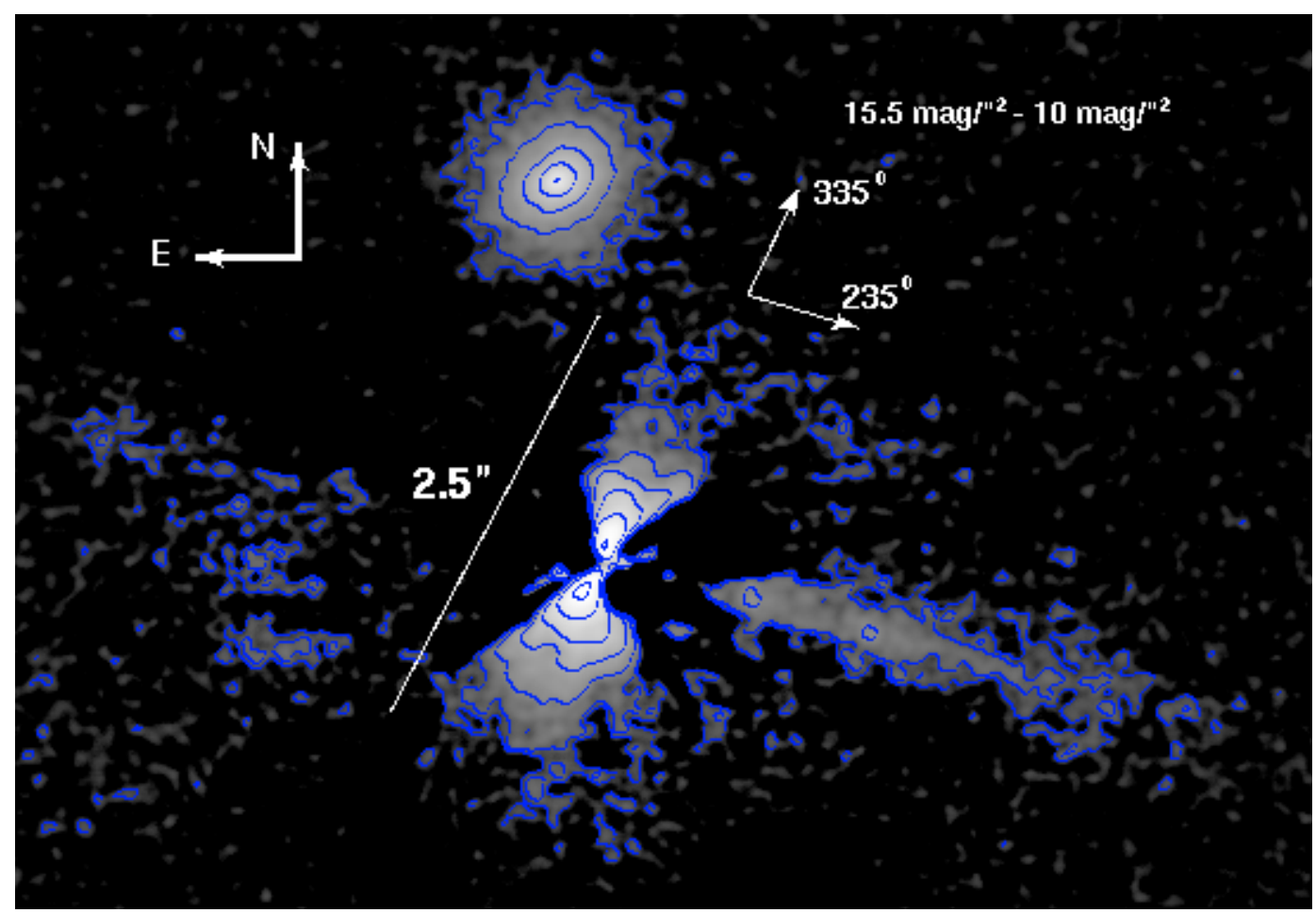

Fig. 4. Final image of PDS 70 after the complete data processing discussed in Sects. 2 and 3. To improve the detection, the final image was smoothed using a Gaussian beam with $F W H M=4$ pixels $=\lambda / d$. The circular disk is seen with an inclination of $62.2^{\circ} \pm 1.6^{\circ}$ and a position angle of $155^{\circ} \pm 2.5^{\circ}$. Perpendicular to the disk, a jet cone is detected in the foreground with a total opening angle of $12^{\circ}$. In the opposite direction of the jet only some residuals are detected. This difference can be explained by the strong absorption of the disk ahead. The scattered image shows only the thin layer of the disk corresponding to $\tau<1$. The brightness scale is logarithmic between levels of 1.125 and 282.72 photons after a proper photometric calibration on the companion. The contour plots show the disk brightness intensity with the values of 10, 11, 12, 13, 14, 15, and $15.5 \mathrm{mag} / \operatorname{arcsec}^{2}$.

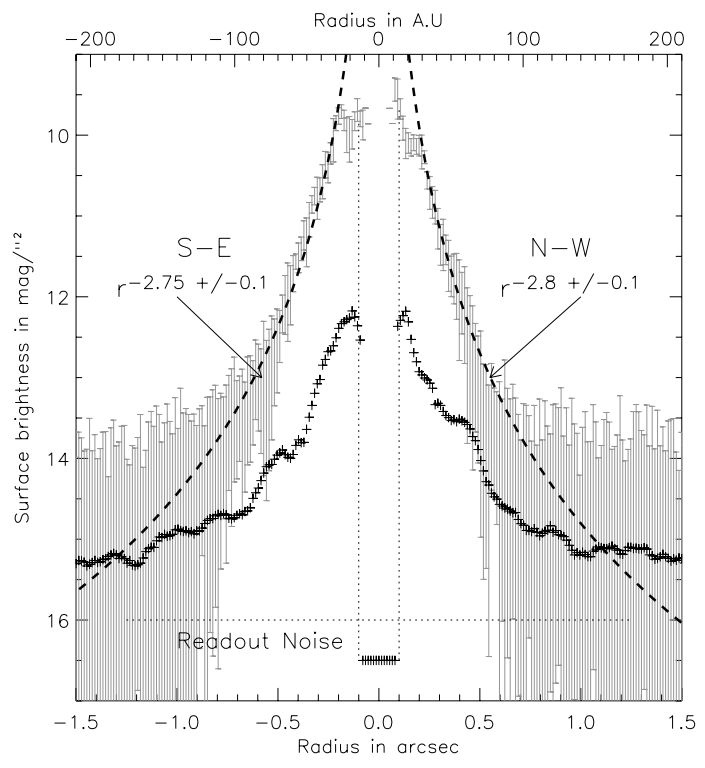

Fig. 5. Surface brightness power law analysis: the figure shows an averaged profile of the disk in a cone of $\pm 10^{\circ}$ around $155^{\circ}$. The dashed line shows the power law fit for the two opposite directions of the disk. The results are $r^{-2.8}(\mathrm{~S}-\mathrm{E})$ and $r^{-2.75}(\mathrm{~N}-\mathrm{W})$ for angular separations between 0.21 to 0 '.55 in radius. The disk profile near the center $r<0$.'21 is flat with an oscillation around 0.15 corresponding to a distance of $20 \mathrm{AU}$. The error bars represent the minimum and the maximum values encountered in the disk. Profiles for distance $r>1$.'25 are readout noise limited. For comparison, we show the averaged profile perpendicular to the main disk orientation, consisting mostly of star light residuals, with plus signs. in a cone of $\pm 10^{\circ}$ around $155^{\circ}$ and gives a fitted power law of $r^{-2.8 \pm 0.1}$ for the S-E direction and of $r^{-2.75 \pm 0.1}$ for the N-W direction. The quoted uncertainties are not due to the photon noise, but rather to the speckle noise near the mask center. The surface brightness peaks at $0.1(\mathrm{~N}-\mathrm{W})$ and $0.19(\mathrm{~S}-\mathrm{W})(14$ and $26 \mathrm{AU}$, respectively) around $10 \mathrm{mag} \operatorname{arcsec}^{-2}$ or $65.7 \mathrm{mJy} \operatorname{arcsec}^{-2}$. The disk brightness falls off more sharply in the $\mathrm{N}-\mathrm{W}$ direction, while presenting a weaker surface brightness. The difference of the disk extension between the S-E and N-W directions is clearly visible in Fig. 4. We also note that the disk brightness seems to flatten with an oscillation inside 30 AU. A large jet in the $\mathrm{E}-\mathrm{W}$ direction $\left(\mathrm{PA}=235^{\circ} \pm 1^{\circ}\right)$ is also detected with angular distances between 0.29 and $4^{\prime \prime}(\approx 41$ to $550 \mathrm{AU})$ with a brightness of $15 \mathrm{mag} \operatorname{arcsec}^{-2}$. Note that the jet orientation and the spider diffraction pattern are angularly separated by only $9^{\circ}$. We also notice that in the opposite direction (E), just a few faint features ( 15.5 to $\left.16 \mathrm{mag} \operatorname{arcsec}^{-2}\right)$ are detected. This observation is compatible with the strong absorption by the thick disk ahead.

Potential planet detectivity. It would be interesting to estimate the detection limit of the NACO/FQPM imaging for young planets as a function of the angular separation in this case. For that, we calculated an azimuthally averaged profile perpendicular to the main disk orientation (see Fig. 5). We then estimated the $5 \sigma$ contrast with respect to PDS 70 (Fig. 6). The luminosity of the putative young planet is calculated with the mass-dependent evolutionary model of Baraffe et al. (2003).

The $K_{\mathrm{s}}$ magnitude of the $10 \mathrm{Myr}$ planet is then computed for masses ranging from 1 to $5 M_{\mathrm{J}}$. Finally, these planet fluxes 


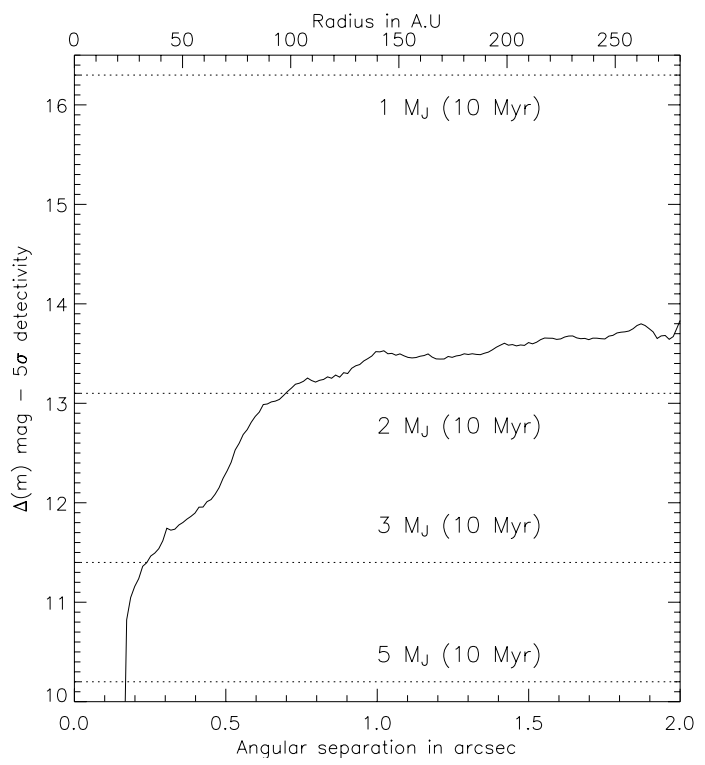

Fig. 6. $5 \sigma$ contrast in $\Delta m$ vs. angular separation in arcsec (bottom) and $\mathrm{AU}$ (top). The solid line represents the detection limit at $5 \sigma$ given by the averaged profile perpendicular to the main disk orientation. The four dotted lines refers to the expected $\Delta M$ of young giant planets for 5 to $1 M_{\mathrm{J}}$. The detection limit is $2 M_{\mathrm{J}}$ at 0.7 and $3 M_{\mathrm{J}}$ at $0{ }^{\prime} .3$.

are compared to the $5 \sigma$ detectivity curve (Fig. 6). The detection limit is $2 M_{\mathrm{J}}$ at 0.7 and $3 M_{\mathrm{J}}$ at 0.3 . These results are to be compared with the detectivity of the spectral differential imaging (SDI) method presented in Masciadri et al. (2005).

\subsection{Thermal infrared data revisited}

The interpretation of the dust emission in young disks relies on models describing how radiation is transferred through them. However, these models are not well constrained because of the lack of observation. Let us then derive some basic characteristics of the disk by revisiting previous mid-infrared SEDs with the new constraints provided by the coronagraphic image. Indeed, the observed disk morphology and orientation help remove the degeneracy of SED modeling.

It is known from previous observations (Metchev et al. 2004; Kessler-Silacci et al. 2005) that the PDS 70 disk presents a large infrared luminosity $\left(L_{\mathrm{IR}} / L_{*}=0.24-0.34\right)$ similar to the TW Hydra stars. This excess corresponds to the thermal reemission of an optically thick disk in response to the central star heating.

SED fitting. Metchev and collaborators came to the conclusion that the PDS 70 circumstellar environment radiates at two different temperatures: $270 \pm 10 \mathrm{~K}$ corresponding to an inner disk of warm dust and $45 \pm 5 \mathrm{~K}$ for an outer component (Metchev et al. 2004) corresponding to a young cold dissipating debris disk. Unfortunately, their SED fit is not precise enough to give further valuable information concerning PDS 70. Therefore, we performed a new fit with the same data (photometry between the $B$ band and $100 \mu \mathrm{m}$ ) plus one data point at $10.7 \mu \mathrm{m}$ obtained with the Long Wavelength Spectrometer at the W.M. Keck Observatory. The $B$ to $I$ photometry was taken from Gregorio-Hetem \& Hetem (2002), while $J$ to $K$ was taken from the 2 MASS catalog (Skrutskie et al. 2006). The $12 / 25 / 60 / 100 \mu \mathrm{m}$ data points were taken from the IRAS catalog

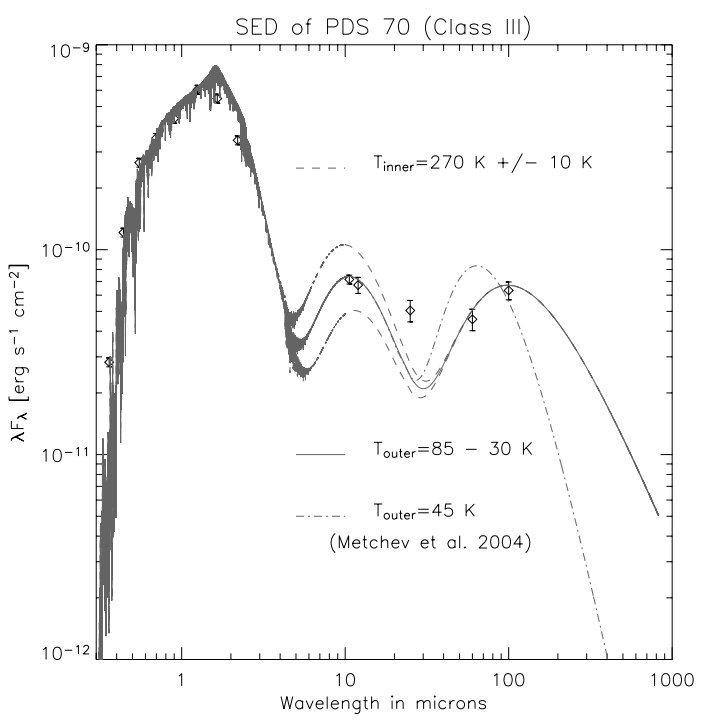

Fig. 7. Measured spectral energy distribution (SED) for PDS 70 compared to that of a reddened NextGen stellar atmosphere model with $T_{\text {eff }}=4400 \mathrm{~K}, \log (g)=4$. The dashed line includes a simple $270 \mathrm{~K}$ blackbody in addition; the dash-dotted line, a $270 \mathrm{~K}$ and a $45 \mathrm{~K}$ blackbody as suggested by Metchev. The continuous line includes the $270 \mathrm{~K}$ component plus a temperature gradient $\left(T \propto r^{-} 0.5\right)$ from $85 \mathrm{~K}(30 \mathrm{AU})$ to $35 \mathrm{~K}(180 \mathrm{AU})$.

(Neugebauer et al. 1988). All photometric data are summarized in Table 3.

A NextGen stellar atmosphere model (Claret \& Hauschildt 2003; Hauschildt et al. 1999) with $T_{\text {eff }}=4400 \mathrm{~K}, \log (g)=4$ was used to fit the stellar flux up to $7 \mu \mathrm{m}$. Then, up to $12 \mu \mathrm{m}$, a stellar spectrum and blackbody with an effective temperature of $270 \mathrm{~K}$ was used to model the inner optically thick disk. A simple cold component at $45 \mathrm{~K}$ gave a strong mismatch with the far infrared IRAS photometry, although the IRAS data is quite accurate and therefore relevant for this target (see Table 2).

To remedy this mismatch, we performed a simulation of the outer disk using the DISKPIC program included in the GENIEsim package (Absil et al. 2006), which simulates the thermal emission from an optically thin debris disk. We assumed that the disk contributing to the far-infrared emission was optically thin and that the temperature varies as $r^{-0.5}$, with $r$ the distance to the star. We then fixed the transition between the inner (optically thick) and outer disk (optically thin) at $\approx 30 \mathrm{AU}$, according to the $K_{\mathrm{s}}$-band observation showing a discontinuity at this particular distance $(\approx 0 . ' 21$, see Fig. 5). Indeed, the surface brightness profile of the disk shows a different power law at short distances, where it seems to be flatter. It is to be noted that only high resolution imaging in the $N$ band could provide the precise temperature variation of the disk.

The main result of this observationally constrained model is that a cold disk with a temperature gradient from $85 \mathrm{~K}$ at $30 \mathrm{AU}$ to $35 \mathrm{~K}$ at $180 \mathrm{AU}$ fits the 60 and $100 \mu \mathrm{m}$ IRAS photometry well. However, concerning the $25 \mu$ m photometry, the simulated flux remained 2 times lower. This difference can possibly be explained by the presence of a strong amorphous silicate emission feature around $20-25 \mu \mathrm{m}$. This emission originates from the Mie scattering of amorphous Olivine and Pyroxene dust grains with sizes between $2 \mu \mathrm{m}$ and $5 \mu \mathrm{m}$ (Kessler-Silacci et al. 2006). The final result of this fit is displayed in Fig. 7. It is to be noted that the stellar atmosphere is affected by a strong reddening $\left(A_{\mathrm{V}}=0.78\right)$. 

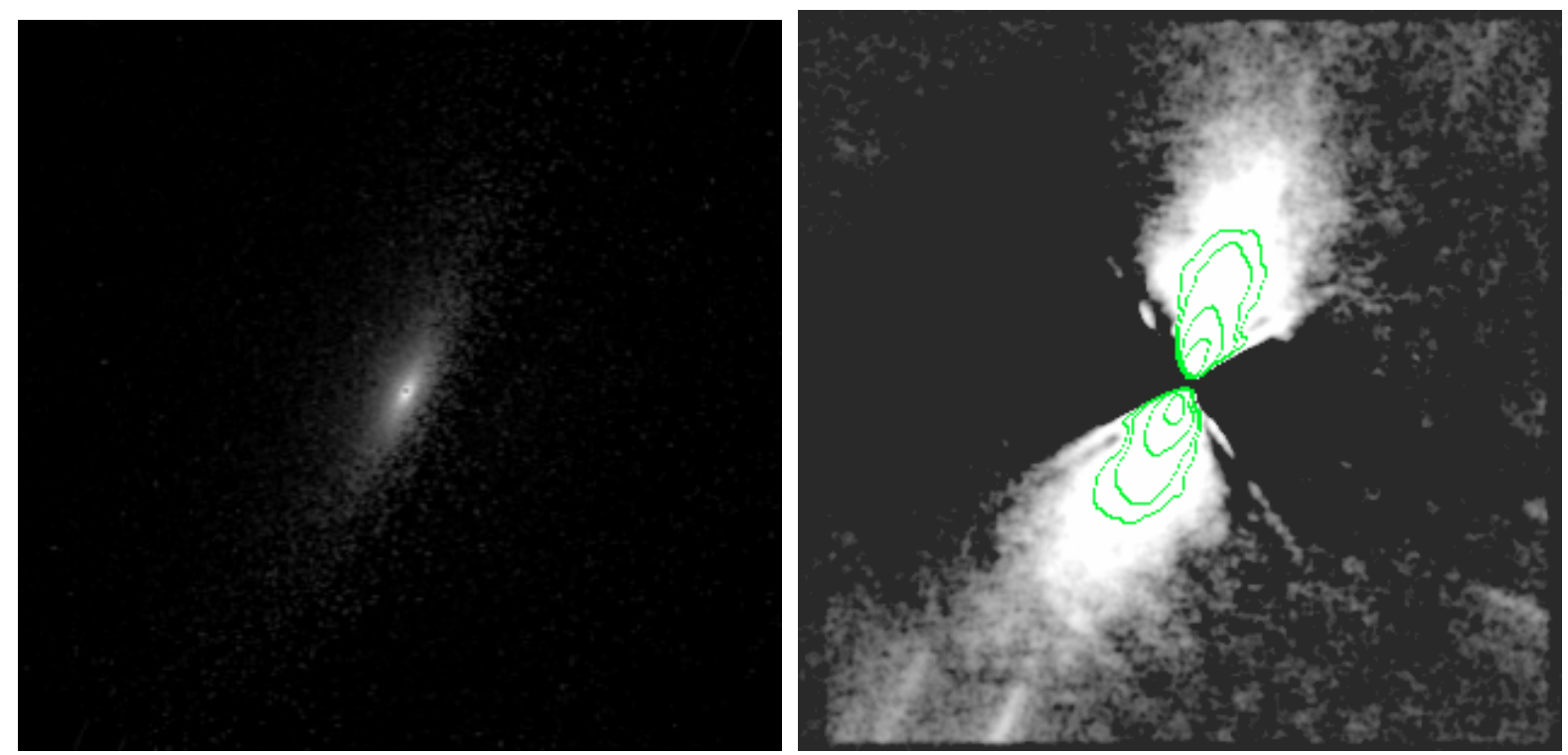

Fig. 8. Left: numerical simulation in $K_{\mathrm{s}}$-band (scattered light) of the PDS 70 disk without PSF convolution. Right: same with PSF convolution and FQPM cross transmission map. For that, we created a synthetic disk and added it to the TTS18 reference star. The image was obtained using the second part of our data reduction algorithm (see Sect. 2.1). The disk orientation was set to $155^{\circ}$ and the inclination to $62^{\circ}$. We also show the contour plots corresponding to 10 to $15.5 \mathrm{mag} / \operatorname{arcsec}^{2}$ brightness scales. We chose a disk mass of $0.001 M_{\odot}$ without the envelope. All images are displayed in logarithmic scale with the same cuts as in Fig. 4.

These new insights on the PDS 70 SED analysis seem to confirm the beginning of the disk clearing process.

Thermal infrared spectroscopy. Kessler-Silacci and collaborators (Kessler-Silacci et al. 2005) have recently presented photometry and spectra in the $N$ band ( 9 to $12 \mu \mathrm{m}$ ). They observed a wide feature for PDS 70 in the $10.2-11.3 \mu \mathrm{m}$ band and explained it by the emission of either various crystalline silicates or amorphous grains of increased sizes. However, the flux in the amorphous Olivine band $\left(9.8 \mu \mathrm{m} / F_{9.8}\right)$ is $2.75 \pm 0.3$ times that of the continuum, whereas the cristalline Forsterite one $\left(11.3 \mu \mathrm{m} / F_{11.3}\right)$ is only $1.68 \pm 0.18$ (ratio $F_{11.3} / F_{9.8}=0.61 \pm$ 0.14 ). The absence of the $11.3 \mu \mathrm{m}$ silicate cristalline feature indicates that the observed broadening is more likely related to grain growth and removal, indicating a possible planetary formation. Indeed, the formation of a cold debris disk is dynamically alleviated by the presence or the formation of giant planets that are ejecting small objects at the periphery of the disk (Moro-Martín \& Malhotra 2005).

\subsection{Modeling the scattered light image}

To model the scattered light image, we used a 3-dimensional scattering code developed by Whitney \& Hartmann $(1992,1993)$ to deduce some information about the disk structure. The three free parameters of the model are the full disk mass $M_{\mathrm{D}}$, the maximum radial extension $r_{\mathrm{D}}$, and the flare angle $\alpha_{\mathrm{f}}$. In addition, the model uses mixed grains with a dust size distribution $n(a) \propto a^{-p}$, where the grain size $a$ ranges from $0.1 \mu \mathrm{m}$ (ISM) to $1 \mathrm{~cm}$ (large grain in a dust disk). Since our observations do not allow us to constrain the exponent $p$ of the power law, we have used a classical dust size distribution where $p=3.5$.

A warm optically thick disk composed of mixed dust, gas, and large grains will naturally flare as a consequence of vertical hydrostatic equilibrium. However, the flare angle parameter does not appear to be critical for the PDS 70 disk since the coronagraphic observation shows only large disk distances $(r>20 \mathrm{AU})$. In fact, our data gives only constraints for the disk mass and its radial extension. The remaining free parameters $M_{\mathrm{D}}$ and $r_{\mathrm{D}}$ are coupled by the disk density, i.e., a larger disk could reproduce the scattered light observation with a lower density. Moreover, in the visible and the near-infrared contributions, stellar radiation penetrates the disk only down to an optical depth of 1 . Due to this limitation, the inferred mass will only be a lower bound (far-infrared or millimeter observations could accuratly give the actual disk mass).

Adding the geometrical constraints given by the coronagaphic image, the disk inclination is fixed to $62^{\circ}$ and the image orientation with respect to the North is $335^{\circ}$. We must also consider image characteristics like sampling (1.858 AU/pixel), convolution by the PSF of PDS 70 without a coronagraphic device, the FQPM orientation, and attenuation in the NACO field of view. Taking all this into account to reproduce the observed geometry of the disk, the model requires a disk radius $r_{\mathrm{D}}$ larger than $500 \mathrm{AU}$ with a total mass $M_{\mathrm{D}}$ between 0.001 to $0.002 M_{\odot}$. We can compare our results for the circumstellar disk around PDS 70 star (a K5 star) with the case of the TW Hydra face-on disk (a K7 star). The same model gives an expected mass of the TW Hya disk of $0.0014 M_{\odot}$ (Weinberger et al. 2002) compatible with our study, whereas the thermal infrared emission model of Calvet et al. (2002) gives a mass of $0.06 M_{\odot}$. This mass discrepancy is representative of the scattered thin disk layer versus the far-infrared / millimeter deeper disk probing as explained before. The disk radius $r_{\mathrm{D}}=500 \mathrm{AU}$ is the minimum disk size that does not induce a large asymmetry of the disk extension, which is not observed in the coronagraphic images.

\subsection{Dynamical considerations}

The transition of the disk profile around $a_{\mathrm{L}} \approx 28-30 \mathrm{AU}$, is likely due to a young giant planet orbiting at $a_{\mathrm{p}} \approx 9.9-10.6 \mathrm{AU}$ in the inner disk. Indeed, circumstellar material exterior to the planet is expected to gain angular momentum from the system and to move away, while material interior to the planet loses 
angular momentum and migrates toward the star. A gap is expected to form if the tidal force overcomes the viscosity. The two main parameters of the tidal interaction are the ratio of the planet and the star masses, $q=M_{\mathrm{p}} / M_{\mathrm{s}}$ and the Reynolds number within the inner disk $\operatorname{Re}$. If $q>1 / \operatorname{Re}$ and $q^{2}<1 / \operatorname{Re}$, a gap should form with the gap edge at the outer Lindblad resonance. The Lindblad resonance can be determined easily from $\Omega_{\mathrm{L}}=\omega_{\mathrm{p}} m /(1+m)$, where $\omega_{\mathrm{p}}=M_{\mathrm{s}}^{1 / 2}(1+q)^{1 / 2} G^{1 / 2} a_{\mathrm{p}}^{-3 / 2}$ is the angular frequency of the orbiting planet, $G$ is the gravitational constant, and $m=1$ for the first Lindblad resonance (Rice et al. 2003a,b). The ratio between the outer Lindblad resonance and planet semi-major axis becomes $a_{\mathrm{L}} / a_{\mathrm{p}}=2^{3 / 2}$ for $m=1$, giving $a_{\mathrm{p}} \approx 10 \mathrm{AU}$ in our case.

\section{Conclusion}

We have discovered an optically thick dust disk around PDS 70, a young star in the Centaurus association (140 pc). The maximum disk extension observed in the coronagraphic image is around $180 \mathrm{AU}$ and presents an important anisotropy in the $\mathrm{S}-\mathrm{E}$ extension. Provided that it is gravitationally linked, a brown dwarf companion (27-50 $M_{\mathrm{J}}$ ) with an effective temperature around $2750 \mathrm{~K}$ has also been detected at about $300 \mathrm{AU}$ from its host star.

The disk anisotropy (S-E) can be explained either by thermal instabilities or by the dynamical effects of the brown dwarf companion. The inner disk (radial distances less than $30 \mathrm{AU}$ ) seems to be flatter. The shape of the SED, the dust grain properties in the thermal infrared, and dynamical considerations are in strong agreement with the formation of a young giant planet at $\sim 10 \mathrm{AU}$, which is currently clearing the inner disk by gravitational tidal force. Indeed, the thermal infrared SED data indicate the prevalence of large amorphous features (all in favor of grain growth and planetary formation) rather than cristalline ones. Finally, our model of the optically thick disk at $270 \mathrm{~K}$ reproduces the scattered light observations. However, the large thermal emission in the far infrared can only be explained by the presence of a young cold debris disk with temperatures between $35 \mathrm{~K}$ and $85 \mathrm{~K}$ at large distances (>30 AU) with respect to the central star. This double disk model fits the PDS 70 SED very well and is corroborated by the numerical model.

Further analysis should be undergone using the $J$ and $H$ bands, polarimetry and SDI observations (Hartung et al. 2004) to infer the inner disk properties. It would also be interesting to image the CO map in millimeter wavelengths with an interferometric array to assess gas dissipation in the disk.
Acknowledgements. P.R. acknowledges the financial support of the University of Liège. D.M. acknowledges the financial support of the Belgian "Fonds pour la formation à la Recherche dans l'Industrie et dans l'Agriculture". O.A. acknowledges the financial support of the "Fond National de la Recherche Scientifique". We thank J.-C. Augereau for the helpful discussions concerning infrared modeling. We thank N. Ageorge, T. Szeifert, and D. Nürnberger for their excellent assistance during the coronagraphic observations on the NACO instrument. The authors are also grateful to the anonymous referee who helped improve the manuscript.

\section{References}

Absil, O., den Hartog, R., Gondoin, P., et al. 2006, A\&A, 448, 787

Baraffe, I., Chabrier, G., Allard, F., \& Hauschildt, P. H. 2002, A\&A, 382, 563

Baraffe, I., Chabrier, G., Barman, T. S., Allard, F., \& Hauschildt, P. H. 2003, A\&A, 402, 701

Boccaletti, A., Riaud, P., Baudoz, P., et al. 2004, PASP, 116, 1061

Boss, A. P. 2002, ApJ, 567, L149

Calvet, N., D'Alessio, P., Hartmann, L., et al. 2002, ApJ, 568, 1008

Chabrier, G., Baraffe, I., Allard, F., \& Hauschildt, P. 2000, ApJ, 542, 464

Claret, A., \& Hauschildt, P. H. 2003, A\&A, 412, 241

Gregorio-Hetem, J., \& Hetem, A. 2002, MNRAS, 336, 197

Haisch, K. E., Lada, E. A., \& Lada, C. J. 2001, ApJ, 553, L153

Hartung, M., Herbst, T. M., Close, L. M., et al. 2004, A\&A, 421, L17

Hauschildt, P. H., Allard, F., \& Baron, E. 1999, ApJ, 512, 377

Kessler-Silacci, J. E., Hillenbrand, L. A., Blake, G. A., \& Meyer, M. R. 2005, ApJ, 622, 404

Kessler-Silacci, J., Augereau, J.-C., Dullemond, C. P., et al. 2006, ApJ, 639, 275

Krist, J. E., Stapelfeldt, K. R., Ménard, F., Padgett, D. L., \& Burrows, C. J. 2000, ApJ, 538, 793

Masciadri, E., Mundt, R., Henning, T., Alvarez, C., \& Barrado y Navascués, D. 2005, ApJ, 625, 1004

Mawet, D., Riaud, P., Baudrand, J., et al. 2006, A\&A, 448, 801

Metchev, S. A., Hillenbrand, L. A., \& Meyer, M. R. 2004, ApJ, 600, 435

Moro-Martín, A., \& Malhotra, R. 2005, ApJ, 633, 1150

Mosqueira, I., \& Estrada, P. R. 2006, Icarus, 180, 93

Neugebauer, G., Habing, H., \& Chester, T. 1988, IRAS Catalogs and Atlases Explanatory Supplement, Vol. 1 (Beichman C.)

Perrin, M. D., Duchene, G., Kalas, P., \& R., G. J. 2006, ApJ, in press

Riaud, P., Boccaletti, A., Rouan, D., Lemarquis, F., \& Labeyrie, A. 2001, PASP, 113,1145

Riaud, P., Boccaletti, A., Baudrand, J., \& Rouan, D. 2003, PASP, 115, 712

Rice, W. K. M., Armitage, P. J., Bonnell, I. A., et al. 2003a, MNRAS, 346, L36

Rice, W. K. M., Wood, K., Armitage, P. J., Whitney, B. A., \& Bjorkman, J. E. 2003b, MNRAS, 342, 79

Rouan, D., Riaud, P., Boccaletti, A., Clénet, Y., \& Labeyrie, A. 2000, PASP, 112, 1479

Schneider, G., Wood, K., Silverstone, M. D., et al. 2003, AJ, 125, 1467

Skrutskie, M. F., Cutri, R. M., Stiening, R., et al. 2006, AJ, 131, 1163

Weinberger, A. J., Becklin, E. E., Schneider, G., et al. 2002, ApJ, 566, 409

Whitney, B. A., \& Hartmann, L. 1992, ApJ, 395, 529

Whitney, B. A., \& Hartmann, L. 1993, ApJ, 402, 605 